\title{
Signal performance of DC-SQUIDs with respect to YBCO thin film deposition rate r $^{\text {is }}$
}

\author{
I. Avcia ${ }^{a}$, B.P. Algul ${ }^{\text {a }}$, R. Akram ${ }^{\text {b,* }}$, A. Bozbey ${ }^{c}$, M. Tepe $^{\mathrm{d}}$, D. Abukay ${ }^{\mathrm{a}}$ \\ a Department of Physics, Izmir Institute of Technology, 35436-Urla, Izmir, Turkey \\ ${ }^{\mathrm{b}}$ Faculty of Engineering Sciences, GIK Institute of Engineering Sciences and Technology, Topi (23640), N.W.F.P, Pakistan \\ c Department of Electrical and Electronics Engineering, TOBB Economics and Technology University, Ankara, Turkey \\ d Department of Physics, Ege University, 35100 Bornova, Izmir, Turkey
}

\section{A R T I C L E I N F O}

\section{Article history:}

Received 18 June 2008

Received in revised form 10 April 2009

Accepted 13 April 2009

Available online 24 April 2009

\section{Keywords:}

YBCO thin films

Deposition rate

Josephson junctions

DC-SQUIDs

\begin{abstract}
A B S T R A C T
The signal performances of $\mathrm{YBa}_{2} \mathrm{Cu}_{3} \mathrm{O}_{7-\delta}(\mathrm{YBCO})$ direct current superconducting quantum interference devices (DC-SQUIDs) have been investigated as a function of the thin film structure affected by the growth process. YBCO thin films of $200 \mathrm{~nm}$ thicknesses were deposited by DC magnetron sputtering using different deposition rates between $1.0 \mathrm{~nm} / \mathrm{min}$ and $2.0 \mathrm{~nm} / \mathrm{min}$ onto $24^{\circ}$ bicrystal $\mathrm{SrTiO}_{3}$ (STO) substrates. The thin film samples were subsequently analyzed by XRD and AFM in order to determine their crystalline structures and surface morphologies respectively. The $67 \mathrm{pH}$ directly coupled DC-SQUIDs with $4 \mu \mathrm{m}$-wide bicrystal Josephson junctions were fabricated, and characterized with respect to their device performances. The variations in the critical current $\left(I_{c}\right)$, the voltage modulation depth $(\Delta V)$ and the noise performance of DC-SQUIDs were reported. The SQUIDs having relatively low deposition rate of $1.0 \mathrm{~nm} / \mathrm{min}$ was observed to have larger voltage modulation depth as well as higher critical current than that of the samples having larger rate of $2.0 \mathrm{~nm} / \mathrm{min}$. The better noise performances were observed as the film deposition rate decreases. The results were associated with the thin film structure and the SQUID characteristics.
\end{abstract}

@ 2009 Elsevier B.V. All rights reserved.

\section{Introduction}

Fabrication of superconducting quantum interference devices (DC-SQUIDs) on high temperature superconducting $\mathrm{YBa}_{2} \mathrm{Cu}_{3} \mathrm{O}_{7-\delta}$ (YBCO) thin films has been intensively studied for last decades in order to establish an appropriate technology essential for high performance devices [1-6]. A number of researches have been focused on the design and fabrication techniques in order to improve the noise performance and peak-to-peak voltage modulation depths of SQUIDs [7-10]. Even though the fabrication complexity of YBCO Josephson junctions has been simplified with bicrystal technology [11-13], the reliability of YBCO based SQUIDs is still the major challenge for many applications such as magneto-cardiography (MCG), susceptometry and non-destructive evaluation (NDE) [14-16,13]. The major limitations of YBCO SQUIDs are their relatively high $1 / f$ noise levels, low output voltage modulation depths $(\Delta V)$ and low stability in time during room temperature storage and thermal cycles $[4,6,17]$. Most of them are related to the structural properties of YBCO thin films and junctions on them. Therefore, the research on the optimization of thin film structure is vital [18,19].

\footnotetext{
is This work was supported by The Scientific \& Technical Research Council of Turkey (under the project numbers of MISAG-264 and MAG-104M194).

* Corresponding author. Tel.: +90 5367075909; fax: +90 3122664579.

E-mail address: rizwanakram75@gmail.com (R. Akram).
}

Critical current density of YBCO thin films depends on the amount of strong pinning sites inside the film structure [20]. The pinning mechanism improves the current flowing capacity by reducing the vortex movement through the superconducting strip line. The pinning sites inside the film structure, which can be formed by adjusting the film growth process, are based on the structural dislocations, fine precipitates with non-superconducting zones and crystalline imperfection. On the other hand, these structural defects have a strong impact on the noise performance of the junctions. Additionally, the surface roughness of the film related to the deposition process is also critical for the junctions' performance.

In order to enhance the noise performance of DC-SQUIDs having identical junctions, the film surface should be very smooth and homogenous along the grain boundary of the bicrystal substrates [21]. The main sources of $1 / f$ noise in SQUIDs are the thermal and critical current fluctuations which are probably related to the homogeneity of the film structure. Another source of $1 / f$ noise is the hopping of flux vortices between the weak pinning sites along the junction strip lines. These are directly related to the growth process of the thin films and the structural formations in the grain boundary region of bicrystal substrate [22].

Besides the film deposition conditions such as growth temperature, oxygen environment, substrate properties and heatingcooling rate, the growth speed is another reason for formation of the structural defects inside the YBCO thin film. It is responsible for the surface roughness as well. Therefore, it may be useful to obtain 
a possible correlation between the SQUID performance and YBCO thin film deposition rate. If the thin film structure and the surface morphology over the GB region can be manipulated by varying the thin film deposition rate at fixed growth temperature, then the primary parameters of the Josephson junctions (the critical current, $I_{\mathrm{C}}$ and the normal state resistance, $R_{\mathrm{n}}$ ) can be controlled, and hence the signal performance of SQUIDs can be optimized. For this reason, we performed a systematic study on the device performance of our DC-SQUIDs by fabricating them onto magnetron sputtered YBCO thin films having different deposition rates between $1.0 \mathrm{~nm} / \mathrm{min}$ and $2.0 \mathrm{~nm} / \mathrm{min}$. The results consisting of the SQUID characteristics, including $I-V$, voltage modulation depths and noise levels, were associated to the thin film structure and surface morphology depending on the film deposition rate.

\section{Device fabrication and optimization}

\subsection{YBCO thin film}

In this study, a number of $200 \mathrm{~nm}$-thick YBCO thin films were deposited by DC magnetron sputtering onto $24^{\circ}(100)$ bicrystal STO substrates. We chose the lower limit of the deposition rate as $1 \mathrm{~nm} / \mathrm{min}$ due to practical reasons such as, the time required to deposit $200 \mathrm{~nm}$ thick YBCO film. We varied the thin film deposition rates between $1.0 \mathrm{~nm} / \mathrm{min}$ and $2.0 \mathrm{~nm} / \mathrm{min}$ at fixed substrate temperature of $780^{\circ} \mathrm{C}$. The thin film growth conditions including argon and oxygen gas pressures, substrate temperature, oxygenation procedure and cooling rates have been used as optimized parameters as follows: the film depositions were carried out at fixed substrate temperature of $780^{\circ} \mathrm{C}$. Argon and oxygen gas pressures were maintained at $0.5 \mathrm{mbar}$ and $0.1 \mathrm{mbar}$, respectively during the deposition. The samples were oxygenated at $600^{\circ} \mathrm{C}$ during $20 \mathrm{~min}$ under $700 \mathrm{mbar}_{2}$ and cooled down to room temperature with the cooling rate of $30^{\circ} \mathrm{C} / \mathrm{min}$. The target-substrate distance was $35 \mathrm{~mm}$. We adjusted the deposition time in order to obtain a fixed film thickness of $200 \mathrm{~nm}$ for all samples. After the deposition, the thin films were first measured as resistance versus temperature in order to characterize their superconducting transition temperatures and transition widths (Table 1 ). Superconducting transition temperatures, $T_{\mathrm{c}}$, of the thin film samples were found as typically $88-89 \mathrm{~K}$ with transition widths of about $0.5 \mathrm{~K}$ from $T_{\mathrm{c} \text {-zero }}$ to $T_{\text {c-onset }}$ as presented somewhere else [19]. However the transition widths of resistance versus temperature characteristics of the $4 \mu \mathrm{m}$ patterned films were about $\sim 1.5 \mathrm{~K}$ which shows a slight degradation in the film quality which can be associated with the wet etching process as shown in Fig. 1a.
Table 1

The deposition parameters, transition temperatures, FWHM values and surface roughness of YBCO thin film samples.

\begin{tabular}{lllll}
\hline Sample number & $\begin{array}{l}\text { Film thickness } \\
(\mathrm{nm})\end{array}$ & $\begin{array}{l}\text { Deposition rate } \\
(\mathrm{nm} / \mathrm{min})\end{array}$ & $T_{\mathrm{c}}(\mathrm{K})$ & FWHM $\left(^{\circ}\right)$ \\
\hline 1 & 200 & 1.0 & 89 & 0.26 \\
2 & 200 & 1.2 & 88 & 0.28 \\
3 & 200 & 1.4 & 89 & 0.29 \\
4 & 200 & 1.6 & 89 & 0.31 \\
5 & 200 & 1.8 & 88 & 0.37 \\
6 & 200 & 2.0 & 88 & 0.46 \\
\hline
\end{tabular}

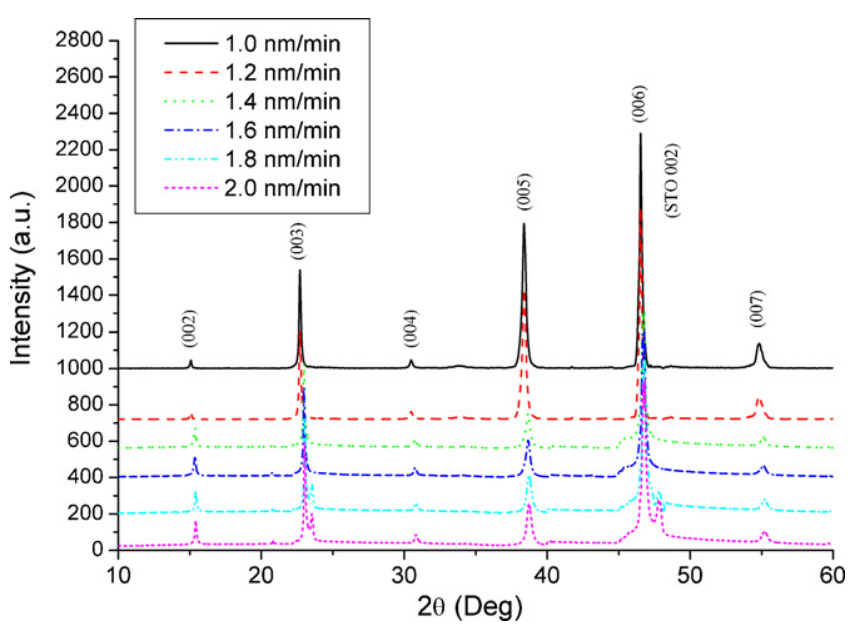

Fig. 2. XRD analysis of YBCO films grown at different deposition rates.

The transitions of all the samples are similar with slight differences which may come from the substrate properties. Since the different substrates purchased from different manufacturers may give rise to different superconducting properties, we purchased all the substrates from the same manufacturer in order to eliminate the additional effects coming from the substrates.

The transition temperatures were observed as similar for all samples and they almost do not depend on the deposition rate as expected. However, the surface morphologies varied with different rates as given as AFM images in Fig. 1b. The mean surface roughnesses of the samples were found to vary from $4 \mathrm{~nm}$ to $10 \mathrm{~nm}$ as the film deposition rate increase from $1.0 \mathrm{~nm} / \mathrm{min}$ to $2.0 \mathrm{~nm} / \mathrm{min}$.

Besides the surface morphology, we performed XRD analysis on the samples and determined their crystalline structures depending on the deposition rate as shown in Fig. 2. The $c$-axis orientations of the structures were determined from FWHM values of $(005)$

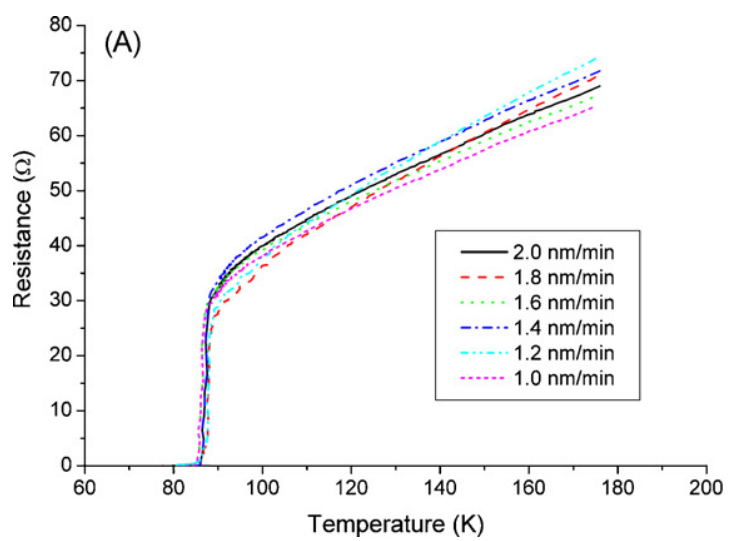

(B)
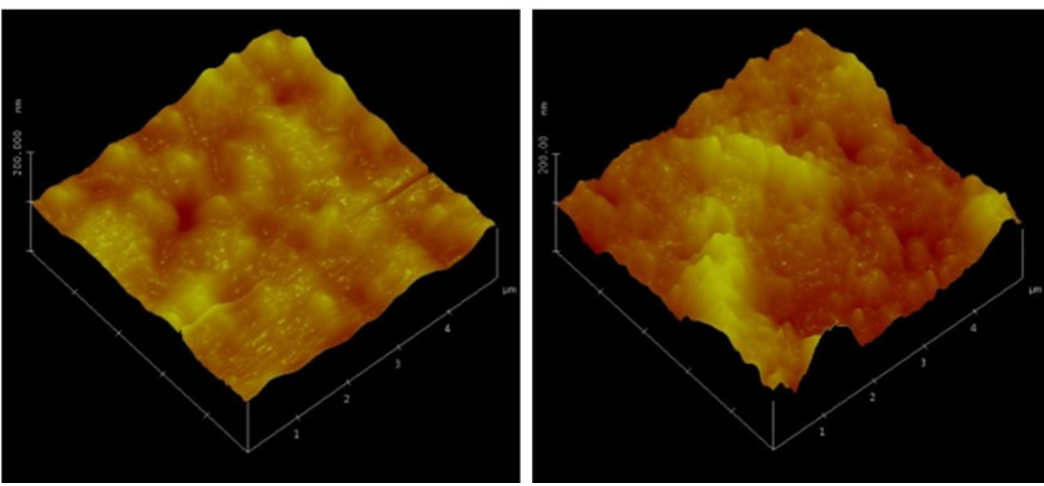

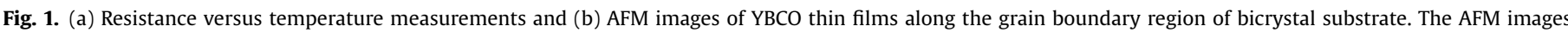
represent the increase in the surface roughness from $4 \mathrm{~nm}$ to $10 \mathrm{~nm}$ as the deposition rates increases from $1.0 \mathrm{~nm} / \mathrm{min}$ to $2.0 \mathrm{~nm} / \mathrm{min}$. 
peaks and we found that the degree of $c$-axis orientation tended to decrease as the deposition rate increases (Table 1 ). We observed some additional peaks of $a b$-orientation in the samples deposited at higher rate. The decrease in the degree of $c$-axis orientation can cause a decrease in the amount of pinning sites parallel to $c$-axis, which makes the structure weaker against the movement of flux vortices.

\subsection{Josephson junctions}

$I-V$ characteristics of the SQUIDs showed significant variation in the critical current values and the normal state resistance depending on the deposition rate as shown in Fig. 3. The $I_{c}$ values decreased from $49 \mu \mathrm{A}$ down to $5 \mu \mathrm{A}$ while the film deposition rate increased from $1.0 \mathrm{~nm} / \mathrm{min}$ to $2.0 \mathrm{~nm} / \mathrm{min}$. It is seen that the $I V$ characteristics of the JJs shown in Fig. 3 are flux flow type rather than RSJ type. We associate this to the wet etching process and DI water rinsing during the fabrication. These both degrade the edges of the YBCO film and would ease the flux entry.

The variations in $I_{\mathrm{c}}$ and $R_{\mathrm{n}}$ values depending on the film growth speed can be explained as the following: as the deposition rate increases, the film structure becomes probably non-homogeneous along the GB line resulting in the difference between the two junctions of the DC-SQUIDs. These non-homogeneous parts can partially consist of either non-superconducting or weak superconducting zones depending on the oxygen content of the film in the $\mathrm{GB}$, which may also become the reason of increase in the normal state resistance of the junctions. The amount of non- and/or weak superconducting parts can differ along the grain boundary, therefore, the Josephson coupling can vary for the junctions. Another reason might be the occurrence of pin-holes or porous structures through the junctions during the high deposition rate. And the last reason can be considered as the partial decrease in the thickness of the film at the edges of the junctions because of high surface roughness at high deposition rate.

\subsection{DC-SQUID fabrication and characterization}

The SQUIDs consisting of directly coupled magnetometer configurations having $4 \mu \mathrm{m}$-wide grain boundary Josephson junctions were patterned by standard lithography and wet etching process. The outer dimensions of magnetometers were designed to have $9 \mathrm{~mm} \times 9 \mathrm{~mm}$ leaving $1 \mathrm{~mm}$ edges on $10 \mathrm{~mm} \times 10 \mathrm{~mm}$ STO sub-
Table 2

Design values of our directly coupled DC-SQUID magnetometers with the YBCO film thickness of $200 \mathrm{~nm}$.

\begin{tabular}{ll}
\hline Parameter & Value \\
\hline$L_{\text {geo, junc }}$ & $8 \mathrm{pH}$ \\
$L_{\text {kin, junc }}$ & $5 \mathrm{pH}$ \\
$L_{\text {geo, wash }}$ & $37 \mathrm{pH}$ \\
$L_{\text {kin, wash }}$ & $17 \mathrm{pH}$ \\
$L_{\mathrm{p}}$ & $4.7 \mathrm{nH}$ \\
$A_{\mathrm{p}}$ & $27 \mathrm{~mm}^{2}$ \\
$A_{\text {eff }}$ & $0.31 \mathrm{~mm}^{2}$ \\
\hline
\end{tabular}

strates to eliminate the edge effects and increase the effective area of the pick-up loop. The pick-up loop includes a square hole placed at the center with one edge of $3 \mathrm{~mm}$. The SQUIDs were designed to have total SQUID loop inductance of $L_{\mathrm{s}} \approx 67 \mathrm{pH}$, pick-up loop inductance of $L_{\mathrm{p}} \approx 4.7 \mathrm{nH}$, pick-up loop area of $A_{\mathrm{p}} \approx 27 \mathrm{~mm}^{2}$, and effective area of $A_{\text {eff }} \approx 0.31 \mathrm{~mm}^{2}$. The total SQUID inductance including the parasitic inductance of junction strip lines was calculated by using the coplanar strip line approximation [23] and the design parameters are given in Table 2 .

The fabricated SQUIDs were placed into chip carriers made by printed circuit board (PCB) material having printed feedback coils. The SQUIDs were then characterized by means of $I-V, V-\Phi$ and noise characteristics regarding the device performance in liquid nitrogen inside a magnetically shielded environment made of a 2-layer $\mu$ metal.

The SQUID output signals are shown in Fig. 4, which indicates variation in the voltage modulation depth $(\Delta V)$ depending on the film deposition rate. $V-\Phi$ measurements were made by flux-locked loop (FLL) electronics inside 2-layer $\mu$-metal shield and the SQUIDs were modulated by 3-turn feedback coil, which was driven with a $20 \mathrm{~Hz}$ saw-tooth waveform having the peak-to-peak amplitude of $1.5 \mathrm{~V}$. In this configuration, we observed the voltage modulation of SQUIDs with countable amount of flux quanta as in Fig. 4. The maximum peak-to-peak voltage modulations $\left(V_{\mathrm{pp}}\right)$ of the SQUIDs tented to decrease from $68 \mu \mathrm{V}$ to $30 \mu \mathrm{V}$ as the film deposition rate increased from $1.0 \mathrm{~nm} / \mathrm{min}$ up to $2.0 \mathrm{~nm} / \mathrm{min}$.

The noise characteristics of DC-SQUIDs were also measured in the frequency range of $1 \mathrm{~Hz}$ to $1 \mathrm{kHz}$. The measurements were performed at bias currents which provide maximum peak-to-peak SQUID voltage modulations. As seen from Fig. 5, the noise levels

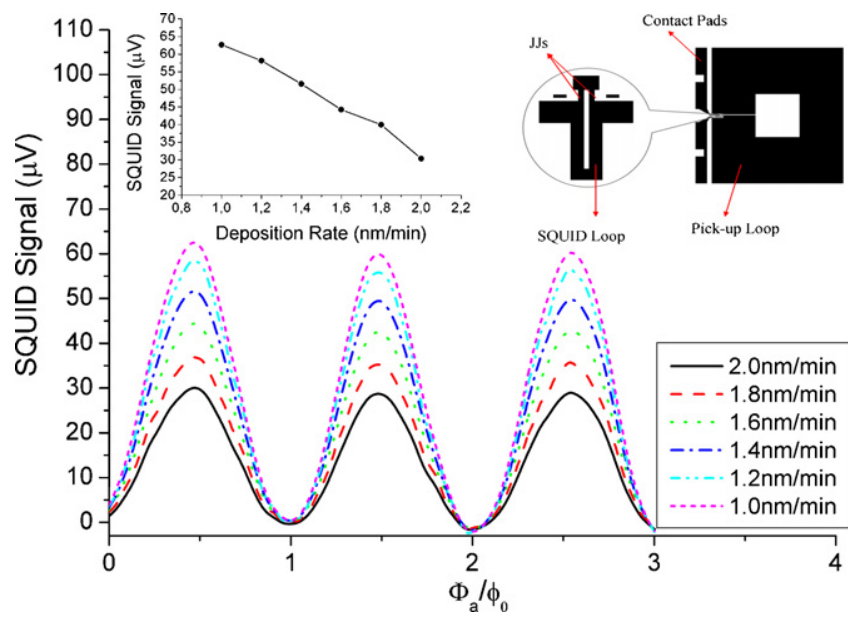

Fig. 4. Output signals of the DC-SQUIDs depending on the film deposition rate. The inset is showing (a) the variation of the SQUID voltage modulation depth depending on the film deposition rate, (b) the layout of the SQUID chip. The SQUIDs were modulated by 3-turn feedback coil by applying $20 \mathrm{~Hz}$ ac signal with $1.5 \mathrm{~V}_{\mathrm{pp}}$.
Fig. 3. $I-V$ characteristics of DC-SQUIDs at $77 \mathrm{~K}$ depending on the film deposition rate. The inset is showing the decrease of the critical current values of SQUIDs as the

film deposition rate increases.
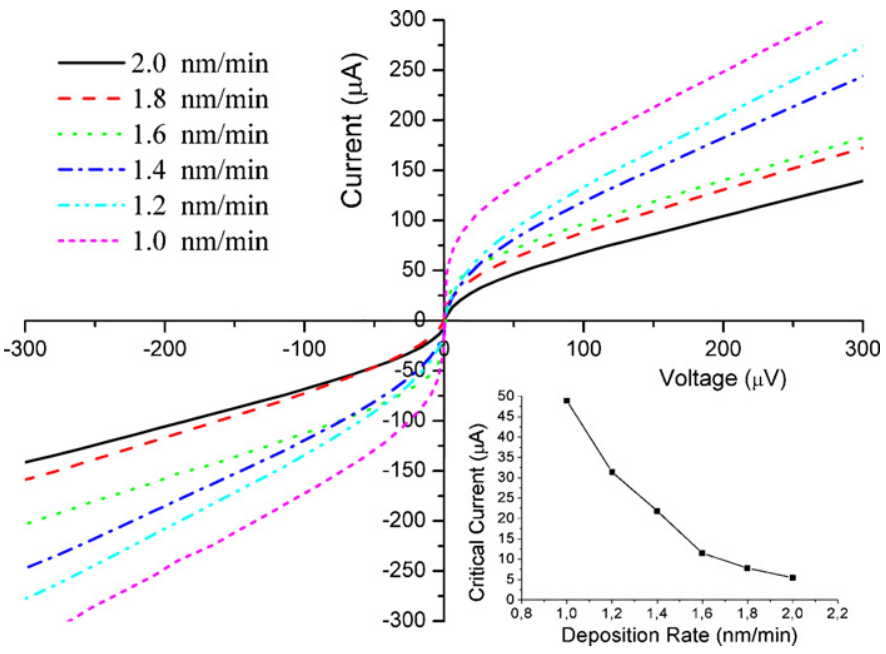

$2.0 \mathrm{~nm} / \mathrm{min}$

$1.8 \mathrm{~nm} / \mathrm{min}$

-.-. $1.4 \mathrm{~nm} / \mathrm{min}$

$\cdots \cdots 1.2 \mathrm{~nm} / \mathrm{min}$

.... $1.0 \mathrm{~nm} / \mathrm{min}$ 


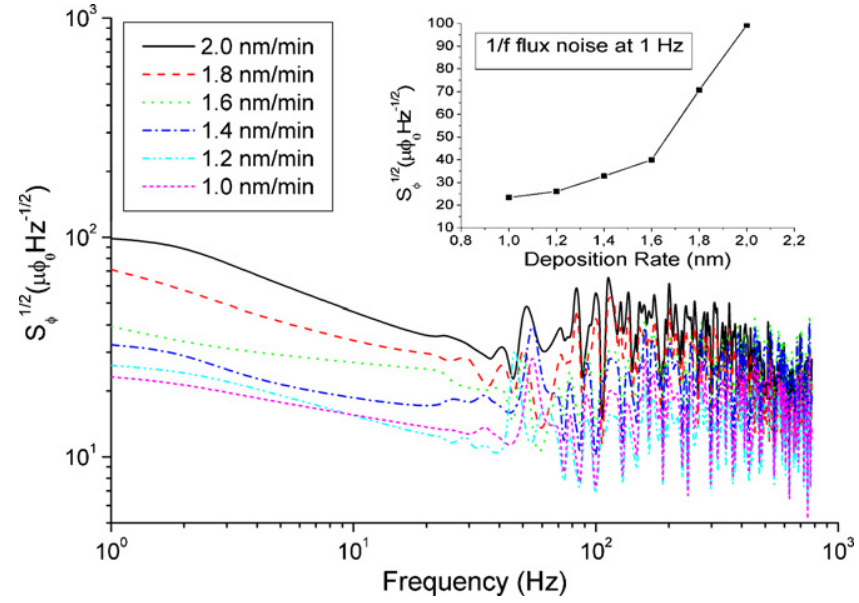

Fig. 5. Noise spectrum of DC-SQUIDs showing flux noises with spectral power densities depending on the film deposition rate. The inset is showing the variation of $1 / f$ noise levels with the rate.

of the SQUIDs tend to increase with the increasing deposition rate. For the SQUID having the lowest deposition rate of $1.0 \mathrm{~nm} / \mathrm{min}$., the white flux and field noises with spectral power densities were found as $12 \mu \phi_{0} \mathrm{~Hz}^{-1 / 2}$ and $80 \mathrm{fTHz}^{-1 / 2}$ respectively at $1 \mathrm{kHz}$. The $1 / f$ field noise was found as $155 \mathrm{fTHz}^{-1 / 2}$ at $1 \mathrm{~Hz}$. As the film deposition rate increased up to $2 \mathrm{~nm} / \mathrm{min}$, the $1 / f$ field noise reached to the level of $663 \mathrm{fTHz}^{-1 / 2}$ at $1 \mathrm{~Hz}$. The increase in the $1 / f$ noise levels with the increasing film deposition rate may depend on; (1) the increase in the non-homogeneity and the surface roughness of the film which can increase the critical current fluctuations in the junctions, (2) the significant decrease in the critical current value which can dominate the effect of thermal noise current, (3) the decreasing of the degree of $c$-axis orientation which can decrease the amount of strong pinning sites along the $c$-axis causing the structure become more available for hopping the flux vortices in the SQUID loop. These may be considered as the sources of high $1 / f$ noise level in the SQUIDs with respect to the high deposition rate of YBCO thin films.

\section{Conclusion}

The improvement on the device performance of YBCO DCSQUIDs with respect to voltage modulation depth $(\Delta V), 1 / f$ noise levels, and stability in time are primarily due the optimization of Josephson junctions. The junction properties such as critical current and normal state resistance as well as the noise characteristics strongly depend on the thin film structure which can be optimized by adjusting the growth process. Therefore, using this fact, we performed an experimental study based on the investigation of the effect of thin film growth process on the signal performance of YBCO DC-SQUIDs. Concerning the variation in the thin film structure and surface morphology depending on the deposition rate, we first characterized the structural properties of YBCO thin films. The $67 \mathrm{pH}$ directly coupled DC-SQUIDs with $4 \mu \mathrm{m}$-wide bicrystal Josephson junctions were fabricated on those thin films and characterized by means $I-V, V-\Phi$ and noise properties. The signal and noise performance of SQUID showed the best results at the lowest deposition rate than that of the high rate of $2.0 \mathrm{~nm} / \mathrm{min}$. This is due to the variation in the structural properties of the thin films. A significant degradation on the SQUID performance was observed as the deposition rate increased. This may be concluded as; the SQUIDs are required high device quality thin films deposited with very controlled deposition procedure and low deposition rate. Such dependence with the SQUID performance and the YBCO thin film deposition process may be applied in addition to the other optimization processes for obtaining high quality and reliable YBCO junction based devices and SQUIDs.

\section{References}

[1] J. Clarke, A.I. Braginski, The SQUID Handbook: Fundamentals and Technology of SQUIDs and SQUID Systems, vol. 1, Wiley-VCH, 2004, pp. 1-28.

[2] R. Kleiner, D. Koelle, F. Ludwig, J. Clarke, Superconducting quantum interference devices: state of the art and applications, Proc. IEEE 92 (2004) 15341548.

[3] H.W. Yu, M.J. Chen, H.C. Yang, S.Y. Yang, H.E. Horng, Effect of the grooved $\mathrm{SrTiO}_{3}$ bicrystal line on the $\mathrm{YBa}_{2} \mathrm{Cu}_{3} \mathrm{O}_{7}$ grain boundary, Physica C 333 (2000) 163-169.

[4] P. Seidel, C. Becker, A. Steppke, M. Buettner, H. Schneidewind, V. Grosse, G. Zieger, F. Schmidl, Long-time stable high-temperature superconducting DC-SQUID gradiometers with silicon dioxide passivation for measurements with superconducting flux transformers, Supercond. Sci. Technol. 20 (2007) S380-S384.

[5] T. Bauch, J. Johansson, K. Cedergren, T. Lindstrom, F. Lombardi, YBCO SQUIDs with unconventional current phase relation, Phys. C: Supercond. 463-465 (2007) 979-984.

[6] M.V. Lyatti, U. Poppe, Y.Y. Divin, Electrical transport and noise properties of $\left[\begin{array}{lll}1 & 0 & 0\end{array}\right]$-tilt $\mathrm{YBa}_{2} \mathrm{Cu}_{3} \mathrm{O}_{7-x}$ grain-boundary junctions with high $I_{\mathrm{c}} R_{\mathrm{n}}$ product, IEEE Trans. Appl. Supercond. 17 (2007) 314-317.

[7] H.H. Qi, P.L. Lang, M.J. Zhang, T.S. Wang, D.N. Zheng, Y.J. Tian, High- $T_{\mathrm{c}}$ DC-SQUID magnetometer and its application in eddy current non-destructive evaluation, Cryogenics 44 (2004) 695.

[8] J. Gao, W.A.M. Aarnink, G.J. Gerritsma, H. Rogalla, Controlled preparation of all high $T_{\mathrm{c}}$. SNS-type edge junctions and DC SQUIDs, Physica C 171 (1990) 126.

[9] M.S. Dilorio, S. Yoshizumi, K.Y. Yang, J. Zhang, M. Maung, Practical high $T_{c}$ Josephson junctions and DC SQUIDs operating above $85 \mathrm{~K}$, Appl. Phys. Lett. 58 (1991) 2552.

[10] R. Simon, et al., Engineered HTS microbridges, IEEE Trans. Magn. 27 (1991) 3209

[11] R. Gross, L. Alff, A. Beck, et al., Physics and technology of high temperature superconducting Josephson junctions, IEEE Trans. Appl. Supercond. 7 (1997) 2929-2935.

[12] H. Hilgenkamp, J. Mannhart, Grain boundaries in high temperature superconductors, Rev. Mod. Phys. 74 (2002) 485.

[13] T. Aytug, M. Paranthaman, K.J. Leonard, K. Kim, A.O. Ijaduola, Y. Zhang, E. Tuncer J.R. Thompson, D.K. Christen, Enhanced flux pinning and critical currents in $\mathrm{YBa}_{2} \mathrm{Cu}_{3} \mathrm{O}_{7-\delta}$ films by nanoparticle surface decoration: extension to coated conductor templates, J. Appl. Phys. 104 (2008), pp. 043906-1-3.

[14] A. Tsukamoto, K. Saitoh, K. Yokosawa, D. Suzuki, Y. Seki, A. Kandori, K. Tsukada, Development of high-throughput fabrication process of HTS SQUID for 51-ch MCG system, Phys. C: Supercond. 426-431 (2005) 1580.

[15] H. Qi, Y. Tian, T. Wang, D. Zheng, A washer type high temperature superconducting DC-SQUID magnetometer with bicrystal junctions on $\mathrm{SrTiO}_{3}$ substrate, Physica C 418 (2005) 23-27.

[16] M.J. Zhang, P.L. Lang, Z.H. Peng, Y.F. Chen, K. Chen, D.N. Zheng, High- $T_{\mathrm{c}}$ planar SQUID gradiometer for eddy current non-destructive evaluation, Chin. Phys. 15 (2006) 1903-1908.

[17] I. Avci, B.P. Algul, A. Bozbey, R. Akram, M. Tepe, D. Abukay, Investigation of the effect of thermal cycling on the device performance of $\mathrm{YBa}_{2} \mathrm{Cu}_{3} \mathrm{O}_{7-\delta} \mathrm{DC}-\mathrm{SQUIDs}$, Supercond. Sci. Technol. 20 (2007) 944

[18] D. Koelle, High transition temperature superconducting quantum interference devices: basic concepts, fabrication and applications, J. Electroceram. 3 (1999) 195-212.

[19] I. Avci, M. Tepe, D. Abukay, Effect of deposition conditions on $\mathrm{YBa}_{2} \mathrm{Cu}_{3} \mathrm{O}_{7-\delta}$ thin films by inverted cylindrical magnetron sputtering and substrate effects, Solid State Commun. 130 (2004) 357-361.

[20] D. Koelle, R. Kleiner, F. Ludwig, E. Dantsker, J. Clarke, High-transitiontemperature superconducting quantum interference devices, Rev. Mod. Phys. 71 (1999) 631.

[21] J. Blomgren, T. Eriksson, E. Tarte, D. Winkler, Noise properties of an YBCOSQUID, Physica C 368 (2002) 191.

[22] M. Kawasaki, P. Chaudhari, A. Gupta, $1 / f$ noise in $\mathrm{YBa}_{2} \mathrm{Cu}_{3} \mathrm{O}_{7-\delta}$ superconducting bicrystal grain-boundary junctions, Phys. Rev. Lett. 68 (1992) 1065.

[23] K. Yoshida, M.S. Hossain, T. Kisu, K. Enpuku, K. Yamafuji, Modeling of kineticinductance coplanar stripline with NbN Thin Films, Japn. J. Appl. Phys. 31 (1992) 3844-3850.

\section{Biographies}

Dr. I. Avci received his PhD degree in 2007 from the Department of Physics at Ege University, Izmir, Turkey. His $\mathrm{PhD}$ research was based on fabrication and applications of YBCO DC-SQUIDs. His current research includes fabrication and testing of HTS and LTS DC-SQUIDs, and Nb/Ta based STJs for low energy X-ray applications in USA.

Ms. B.P. Algul received her BSc degree in physics from Dokuz Eylul University in 2004 and MSc degree from Izmir Institute of Technology, Izmir, Turkey in 2008. She 
is currently working towards PhD degree at the Physical Electronics Departments, Tokyo Institute of Technology, Tokyo, Japan.

Dr. R. Akram received his BS degree in Electrical and Electronics Engineering from Eastern Mediterranean University, North Cyprus in 1997. He has completed his MS in 2000 from Bilkent University, Ankara, Turkey and research was focused on HTSC Bolometers design and characterization. He has received his PhD degree in 2005 from Electrical and Electronics Engineering Department at Bilkent University, Ankara, Turkey. His PhD research was based on the fabrication, optimization and application of rf-SQUID magnetometers and gradiometers and their application in scanning SQUID microscopy and non-destructive evaluation systems. He has been visiting forschungszentrum Juelich from 2000 to 2003 as guest scientist working on a joint project from Research council of Turkey and Germany. He has been associated with Izmir Institute of technology, Izmir, Turkey from 2005 to 2006 as post-doctoral research fellow working on HTSC thin film fabrication and optimization for DCSQUIDs and the development of NDE system. In 2006-2008 he has been associated with advance research lab at Bilkent University, Ankara, Turkey as post-doctoral research fellow working on optimization of scanning Hall probe microscopy and micro-Hall sensors for variable temperature applications. In August 2008 he joined Ghulam Ishaq Khan Institute of Engineering Sciences and Technology, Topi, Pakistan as assistant professor in the Department of Engineering Sciences. His research interest includes high temperature superconducting electronic devices. Applications of superconducting technology, HTSC thin film fabrication and optimization, fabrication of micro- and nano-Hall effect sensors and magnetic imaging systems.
Dr. A. Bozbey received his BS, MS, and PhD degrees in electrical and electronics engineering from Bilkent University, Turkey, in 2001, 2003, 2006 respectively. In 2002 he worked as a guest researcher at Julich Research Center, Germany and in 2007 he worked as a post-doctoral researcher at Nagoya University, Japan. In 2008 he joined the Electrical and Electronics Engineering Department, TOBB Economy and Technology University, Turkey, where he teaches in the areas of solid-state, and electronics, while supervising the Superconductivity Electronics Research Laboratory. His research interests include the design and modeling of high-temperature superconducting infrared detectors, SQUID fabrication and applications, and rapid single flux quantum based circuit design.

Dr. M. Tepe received his BSc and PhD degrees in physics in 1989 and 1997 respectively from Ege University, Izmir, Turkey. His PhD thesis is on the experimental study of dopant effect in high- $T_{\mathrm{c}}$ superconducting oxides. He is an associate professor since 2004 at Ege University Physics Department. His main research areas are bulk high$T_{\mathrm{c}}$ superconductors, high- $T_{\mathrm{c}}$ superconducting thin films and sensors based on these materials.

Dr. D. Abukay received his BSc degree from Physics Department at METU, Ankara, Turkey in 1970, and PhD from Ege University, İzmir, Turkey in 1976, He became professor in 1989 at Ege University in the Department of Physics, at present he is employed at Izmir Institute of Technology, Science Faculty, Physics Department (since 2002) and his current field of interest includes superconductivity, superconducting devices, and thin films. 